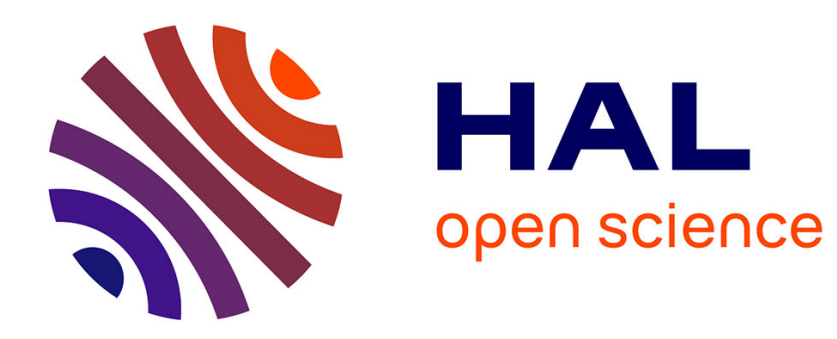

\title{
À propos de l'ouvrage de David A. Lax et James K. Sebenius The Manager as Negotiator
}

Sophie Allain

\section{To cite this version:}

Sophie Allain. À propos de l'ouvrage de David A. Lax et James K. Sebenius The Manager as Negotiator. Négociations, 2004, 2, pp.135-138. 10.3917/neg.002.135 . hal-01122978

\section{HAL Id: hal-01122978 \\ https://hal.science/hal-01122978}

Submitted on 31 Mar 2015

HAL is a multi-disciplinary open access archive for the deposit and dissemination of scientific research documents, whether they are published or not. The documents may come from teaching and research institutions in France or abroad, or from public or private research centers.
L'archive ouverte pluridisciplinaire HAL, est destinée au dépôt et à la diffusion de documents scientifiques de niveau recherche, publiés ou non, émanant des établissements d'enseignement et de recherche français ou étrangers, des laboratoires publics ou privés. 


\title{
À PROPOS DE L'OUVRAGE DE DAVID A. LAX ET JAMES K. SEBENIUS THE MANAGER AS NEGOTIATOR
}

\author{
Sophie Allain
}

De Boeck Université | Négociations

2004/2 - no 2

pages 135 à 139

ISSN 1780-9231

Article disponible en ligne à l'adresse:

http://www.cairn.info/revue-negociations-2004-2-page-135.htm

Pour citer cet article :

Allain Sophie, «À propos de l'ouvrage de David A. Lax et James K. Sebenius The Manager as Negotiator », Négociations, $2004 / 2$ no 2, p. 135-139. DOI : 10.3917/neg.002.135

Distribution électronique Cairn.info pour De Boeck Université.

(c) De Boeck Université. Tous droits réservés pour tous pays.

La reproduction ou représentation de cet article, notamment par photocopie, n'est autorisée que dans les limites des conditions générales d'utilisation du site ou, le cas échéant, des conditions générales de la licence souscrite par votre établissement. Toute autre reproduction ou représentation, en tout ou partie, sous quelque forme et de quelque manière que ce soit, est interdite sauf accord préalable et écrit de l'éditeur, en dehors des cas prévus par la législation en vigueur en France. II est précisé que son stockage dans une base de données est également interdit. 


\section{À propos de l'ouvrage de David A. Lax et James K. Sebenius The Manager as Negotiator}

Sophie Allain

INRA, ENS Cachan / GAPP-CNRS

Paru en 1986, The Manager as Negotiator (The Free Press, New York) s'est rapidement imposé comme un classique de la négociation, tant dans le monde académique qu'auprès des décideurs publics ou privés. L'originalité de cet ouvrage réside en effet dans l'analyse approfondie de la négociation à des fins prescriptives qu'il propose aux décideurs, quel que soit leur domaine d'activités et que celui-ci mette en jeu explicitement des négociations ou non. En effet, pour David A. Lax et James K. Sebenius, à la fois enseignants et consultants, la négociation représente le cœur de l'activité managériale et maints problèmes organisationnels proviennent d'une mauvaise compréhension et d'un traitement inapproprié de ce type de processus. Pour être susceptible d'aider les « négociateurs », c'est-à-dire tout acteur impliqué dans une situation de négociation, les auteurs considèrent qu'il convient cependant d'éviter deux écueils fréquents dans la littérature: les descriptions analytiques qui ne font que découvrir qu'il existe des négociations, et les approches prescriptives qui se fondent sur une conception biaisée, voire idéologique, de la négociation, celle-ci n'étant vue que comme un processus purement coopératif (approche win-win) ou purement compétitif (approche win-lose). Aussi leur objectif est-il de construire une grille d'analyse de la négociation qui tienne compte de la complexité de ce processus, et notamment de la tension fondamentale entre mouvements coopératifs et mouvements compétitifs, inhérente à toute négociation. Dans cette entreprise, ils se réclament de deux influences : d'une part, des réflexions issues de la théorie des jeux menées par des auteurs comme Th. Schelling et H. Raïffa, et d'autre part, des travaux menés en management et politique des organisations, en particulier ceux de R. Neustadt, G. Allison, J. Bower et M. Moore.

L'ouvrage s'organise en trois parties:

- Une partie introductive cherche à définir ce qu'est une négociation et à montrer le caractère général de ce type de processus dans toute activité managériale.

Pour Lax et Sebenius, toute négociation repose sur les éléments-clés suivants : 
- l'interdépendance, qui implique des limites à ce que chacun peut faire individuellement et qui donne un intérêt à une action conjointe;

- la perception d'un conflit, ou du moins d'une divergence de préférences;

- une interaction stratégique, au sens où chacun définit ses actions en tenant compte de celles des autres et se soucie de la défense de ses propres intérêts;

- la possibilité d'un accord.

Une négociation est alors définie comme « un processus d'interaction potentiellement stratégique, dans lequel deux parties ou plus en conflit cherchent à faire mieux qu'elles ne pourraient le faire autrement à travers une action décidée conjointement ».

Ces caractéristiques font de la négociation un processus central de la vie sociale et notamment de l'activité managériale, bien que cela soit davantage perçu comme évident dans les situations où il s'agit de traiter avec l'« extérieur » qu'à l'« intérieur » même d'une organisation. Or, les approches managériales appréhendent mal ce type d'activité : en effet, la conception traditionnelle du management en terme d'autorité et de contrôle tend à oublier que toute forme d'autorité repose sur une négociation préalable, comme à négliger la marge d'autonomie dont dispose tout acteur; par ailleurs, les conceptions purement coopératives (telles que celles prônées par le mouvement des relations humaines, les approches en termes de leadership, de management participatif, ou encore de culture organisationnelle) ne tiennent pas compte des conflits d'intérêts et des perceptions divergentes existants dans toute organisation. Les auteurs attribuent la résistance fréquente à reconnaître la généralité des situations de négociation à la remise en cause de l'autorité et à la légitimation des différences qu'une telle position implique; de ce fait, des situations réelles de négociations sont souvent définies comme de simples situations de résolution de problème où les conflits sont masqués.

- La première partie vise à présenter la construction d'une grille d'analyse de la négociation, qui articule trois dimensions : statique (a), dynamique (b) et « évolutionniste » (c).

(a) Pour Lax et Sebenius, toute négociation repose sur les trois éléments de base suivants:

- les « intérêts » en jeu, qui constituent la base de la négociation et en donnent la mesure;

- les « alternatives » à la recherche d'un accord, qui fournissent les limites de la négociation;

- les « accords » potentiels.

Sur le plan prescriptif, les auteurs préconisent notamment:

- d'analyser soigneusement les intérêts sous-jacents en jeu, en ne s'arrêtant pas seulement aux positions affichées. S'ils rejoignent sur ce point Fisher et Ury $(1981)^{1}$, ils refusent par contre d'en faire une règle géné-

1 Fisher R., Ury W., (1981), Getting to yes. Houghton Mifflin Company, Boston. 
rale comme ces derniers, en expliquant que dans des situations de conflits de valeurs, il est au contraire préférable de se concentrer sur des questions correspondant à des enjeux limités; pour clarifier les intérêts qui sont souvent flous, ils conseillent de leur attribuer des valeurs permettant de les classer.

- d'évaluer les alternatives à la négociation, en ayant éventuellement recours à un avis neutre ou en testant le point de vue de l'autre partie, du fait de la tendance à surestimer ses propres alternatives. L'« immaturité » d'une situation de négociation serait ainsi fondamentalement liée à des estimations trop optimistes des alternatives de part et d'autre.

(b) Si ces trois éléments mettent en évidence la «configuration » de la négociation, c'est la dynamique de « création de valeur » (creating value) et de « lutte pour l'appropriation » (claiming value) qui permet de rendre compte de la fabrication du résultat:

- la notion de création de valeur repose sur la recherche de gains mutuels et renvoie à trois types de résultats : un accord offrant des possibilités supérieures à celles de ses alternatives, un accord meilleur qu'un autre, et la possibilité de faire davantage ensemble que cela n'était supposé (jeu à somme positive). À la différence de la plupart des auteurs qui cherchent à définir le style de négociation favorisant la création de valeur (attitude d'ouverture, partage d'informations, créativité. ..), Lax et Sebenius s'attachent à comprendre les ressorts de ce processus: ils mettent ainsi en évidence le rôle-clé des différences d'intérêts ou de perceptions, qui permettent de jouer sur des complémentarités; des intérêts partagés (maintien de la relation ou valeurs supérieures communes); et des économies d'échelle.

- la lutte pour l'appropriation vise, elle, la recherche du gain maximal au détriment de l'autre (jeu à somme nulle). Elle passe en particulier par des actions sur l'environnement (manipulation de la perception des alternatives ou des aspirations de l'autre partie...) et par différentes tactiques comme les engagements (commitments) visant à convaincre l'autre que l'on est arrivé au point ultime d'une négociation, les prises de positions, et différents attitudes (dissimulation d'informations, menaces...).

La négociation comporte une tension essentielle entre ces deux types de mouvements, qui peuvent s'exprimer tant au plan tactique que stratégique. Ceux-ci sont néanmoins exacerbés au plan tactique, parce que les approches cherchant à créer de la valeur sont sensibles aux approches visant à se l'approprier et que ces dernières peuvent même empêcher la création de valeur. Cette tension constitue le « dilemme du négociateur » qui peut être géré à titre individuel ou à l'échelle du processus dans son ensemble (par des techniques comme la médiation, la formulation progressive d'un texte requérant l'approbation des parties, 
ou la séparation entre phase d'invention et phase de décision, par exemple).

(c) Enfin, le « jeu » lui-même peut-être modifié, à travers le choix des questions traitées, des parties impliquées et des intérêts mis en exergue. Cette troisième dimension prend un sens vis-à-vis de deux types de questions : quand et comment changer le jeu au cours d'une négociation? Comment configurer le jeu pour favoriser le déroulement d'une négociation dans un certain sens?

- La seconde partie, plus limitée, applique la grille d'analyse précédente à des questions concrètes de management.

En effet, un décideur doit fondamentalement se soucier :

- de son « mandat », c'est-à-dire de la combinaison des buts à atteindre, de l'autorité et des ressources attribuées, ainsi que des conditions et attentes afférentes;

- de la «production » permettant de satisfaire ce mandat, que celle-ci soit effectuée directement ou indirectement;

- de la « stratégie » permettant d'intégrer le tout.

Le mandat comme la production mettent en jeu différents types de négociations que Lax et Sebenius passent en revue. Ceux-ci insistent en particulier sur l'importance de construire une coalition pour soutenir un mandat, et portent une grande attention aux situations de management indirect dans lesquelles l'autorité et les ressources sont réparties alors que la responsabilité est concentrée.

Ils en concluent que le manager se trouve toujours « au milieu », entre des négociations internes et externes, et qu'il a intérêt à se comporter en «double agent», en utilisant les unes pour influencer les autres et réciproquement; à ce titre, il peut être vu comme un « médiateur » d'un type particulier.

Au total, cet ouvrage s'adresse à un large public:

- les chercheurs y trouveront une analyse riche et subtile de la négociation et pourront notamment comparer le modèle de Lax et Sebenius aux modèles intégrés antérieurs de la négociation ${ }^{2}$ proposés par Sawyer et Guetzkow $(1965)^{3}$ et Walton et McKersie (1965) ${ }^{4}$;

- les praticiens (négociateurs professionnels et décideurs de tout domaine) apprécieront son caractère pédagogique, ainsi que l'abondance et la variété des exemples.

2 Voir Dupont Ch., (1994), La négociation. Conduite, théorie, applications. Dalloz, Paris.

3 Sawyer J., Guetzkow H., (1965), "Bargaining and Negotiation in International Negotiations". In Kelman H., International Behavior. Holt, Rinehart et Winston.

4 Walton R. E., McKersie R. B., (1965), A Behavioral Theory of Labor Negotiations. ILR Press, Ithaca, New York. 